\title{
An Implicit-Semantic Tag Recommendation Mechanism for Socio-Semantic Learning Systems
}

\author{
Paul Seitlinger ${ }^{1}$, Tobias Ley ${ }^{2}$, and Dietrich Albert ${ }^{1}$ \\ ${ }^{1}$ Knowledge Management Institute, Graz University of Technology, Austria \\ \{paul.seitlinger, dietrich.albert\} atugraz.at \\ ${ }^{2}$ Center for Educational Technology, Tallinn University, Estonia \\ tley@tlu.ee
}

\begin{abstract}
In recent years Social Tagging (ST) has become a popular functionality in social learning environments, not least because tags support the exchange of users' knowledge representations, a process called social sensemaking. An important design feature of ST-Systems (STS) is the tag recommendation service. Several principles for tag recommendation mechanisms (TRM) have been proposed, which are built upon a technical and statistical perspective on STS and based on aggregated user data on a word level. Up to now, a cognitive perspective also taking into account memory processes has been neglected. In this paper we therefore introduce a TRM that applies a formal theory of human memory to model a user's prototypical tag configurations. The algorithm underlying the TRM is supposed to recommend psychologically plausible tag combinations and to mediate social sensemaking.
\end{abstract}

Keywords: Tagging, Categorization, Cognitive Modelling, MINERVA2, Tag-Recommendation-Algorithm.

\section{Introduction}

In recent years, Social Tagging (ST) has become a popular functionality in the Web allowing people to freely associate textual labels (called tags) to resources. Prominent ST-Systems (STS) are http://del.icio.us (social bookmarking platform) or http://flickr.com (photo sharing platform), which we regard as socio-semantic learning environments. Dynamic interactions between representations on an external level (tags and resources) and semantic memory processes on an internal level (categorization) expedite social sensemaking [1], i.e. cooperative categorization and indexing of Web resources. To mediate these social learning processes we need services that analyze statistical structures on the word level and are embedded into a cognitivepsychologically plausible framework.

With respect to its usefulness for educational activities, empirical studies of Kuhn et al. (e.g. [5]) give evidence that ST supports an important aspect of science education in schools and university courses, namely reflecting on the utility of data and annotating this reflection for later recall. A design recommendation of [5] is that teachers or lectors deploying ST for social learning processes should provide a schema for the tagging activity and should categorize tags in a relevant way. 
In this paper we introduce the principles of a tag-recommendation mechanism (TRM), which is motivated by empirical studies [6,6] and built upon MINERVA 2 [3], a formal theory of human memory. This TRM is designed to extract prototypical tag combinations (so called gist traces) from a user's tagging behavior and to suggest tags in a categorized and psychologically meaningful way. The suggestion of gist-traces is supposed to give a supportive schema during the tagging activity. Beyond that, it is conceived to identify and recommend users with similar gist traces, thereby mediating social sensemaking.

The structure of this article is as follows. First, we provide a brief overview of previous TRMs (section 2.1). Second, we briefly summarize some cognitivepsychological work on STS to motivate the principles of our TRM and briefly describe MINERVA2 (section 2.2). Third, we provide simple equations to derive appropriate tag recommendations (sections 2.3 and 2.4).

\section{An Implicit-Semantic Tag Recommendation Mechanism}

\subsection{Previous Tag Recommendation Mechanisms}

Referring to [2] there are currently four different approaches to design TRMs. One approach is the analysis of tag quality, e.g. its popularity and semantic distinctiveness to other tags. A second approach is the computation of tag co-occurrence to gather similarities between pairs of tags for the recommendation of appropriate tag combinations. The third approach relies on mutual information between words, documents and tags. One example is collaborative filtering for recommending tags in folksonomies [4]. For a given user a neighborhood is formed consisting of users with similar tag or resource collections. Tags frequently occurring within the neighborhood are then recommended. The fourth approach takes into account the content of a resource and ranks tags according to their relevance to the resource's content. [4] applied an adapted PageRank algorithm, which ranks the importance of vertices (tags, users, resources) as a function of their edge degrees. The most dominant approach simply counts the number of tag occurrences and suggests the most popular ones.

All these approaches are based on aggregated user data and - to some extent - on the "wisdom of the crowd". However, they abstract from users' preferences and neglect their typically verbal categorization behavior. Cognitive-psychological studies (e.g. $[1,6,6])$, briefly described in the next sub-section, show that these approaches would benefit from mechanisms applying formal theories of human semantic memory. Such an extension would help to realize the suggestion of [5] to provide a categorical schema for the tagging activity during educational tasks.

\subsection{Theoretical and Empirical Background}

[1] provided a formal model of human categorization in STS. They put emphasis on implicit (automatic) categorization processes of a user during a tag-based inference of a resource's gist (topic) as well as during gist-based tag-assignments. By means of a multinomial model of ST [6] and [6] empirically showed that implicit categorization 
processes (gist-based reconstructions) are indeed in play during the generation of tags. More precisely, users retrieve an implicit gist-trace from their semantic memory to reconstruct the meaning of previously perceived tags. Afterwards, tags are chosen to index the implicit gist-trace. Here, we introduce an implicit-semantic tagrecommendation mechanism (isTRM) that mimics the gist-based reconstruction process investigated by [6].

As described above, the isTRM is built upon MINERVA2 [3] that formally describes implicit, reconstructive processes triggered by stimuli (e.g. words or tags). The general assumption is that a stimulus (e.g. the word "bird") strongly activates traces (internal representations) in semantic memory, which share many features with the stimulus (e.g. sparrow, raven, falcon, etc.); all other traces stay relatively dormant (e.g. different dog exemplars). All the features common across the activated traces (e.g. feathers, wings, etc.) constitute the concept that comes into mind. The outcome of this activation process is a prototype or gist: an abstract representation of all single traces activated by the stimulus (e.g. a prototypical bird). MINERVA2 provides a formalization of this reconstructive process. Memory traces as well as stimuli are formalized as vectors where feature values $(-1,1)$ encode the existence/nonexistence of features. Thus, the semantic memory is represented as a matrix (a set of row vectors). A particular algorithm (see 2.4), which multiplies the matrix by a stimulusvector, yields a content-vector displaying the prototype.

We draw on the MINERVA2 notations to represent a user's tag assignments (TAS for short) in form of vectors, whose feature values encode the assignment/nonassignment of a tag to a particular resource, and on the MINERVA2 algorithm to extract the user's prototypical tag combinations.

\subsection{Notation of a User's Personomy}

The basis of the isTRM is the formalization of a user's semantic traces left in the STS, which are verbalized in form of her or his tag assignments (TAS). To define a TAS we refer to [4] and represent an STS as a triple of the finite sets $U, T$ and $R$, whose elements are the users, tags and resources, respectively. There exists a ternary relation $Y$ between the three sets, i.e. $Y \in U \times T \times R$., and the TAS $(u, t, r)$ are the elements of $Y$. The collection of all TAS of user $u_{i}$ is called personomy [4]; the collection of all personomies constitutes the folksonomy.

For $m$ resources and $n$ tags of the whole folksonomy, we notate the personomy of a user $u_{i}$ in a resource-tag matrix $\mathbf{X} \in\{-1,1\}^{m \times n}$ that can be divided into row vectors: $\mathbf{X}$ $:=\left[\vec{x}_{1}, \ldots, \vec{x}_{m}\right]$ with $\vec{x}_{r}:=\left[x_{r 1}, \ldots x_{r n}\right]$, for $r:=1, \ldots, m$. We call $x_{r t}$ a tag-feature indicating that a user assigned tag $t$ to the resource $r$, and $x_{r t} \in\{-1,1\}$. Thus, each row vector represents a particular TAS of a user $u_{i}$ that we call semantic trace. The middle part of Fig. 1 schematically presents this resource-tag matrix $\mathbf{X}$. For instance, the first tag-feature of the semantic trace $\vec{x}_{1}$ indicates that the user assigned the tag "memory" to the resource $r_{1}$; the second tag-feature represents the non-assignment of the tag "Java". 


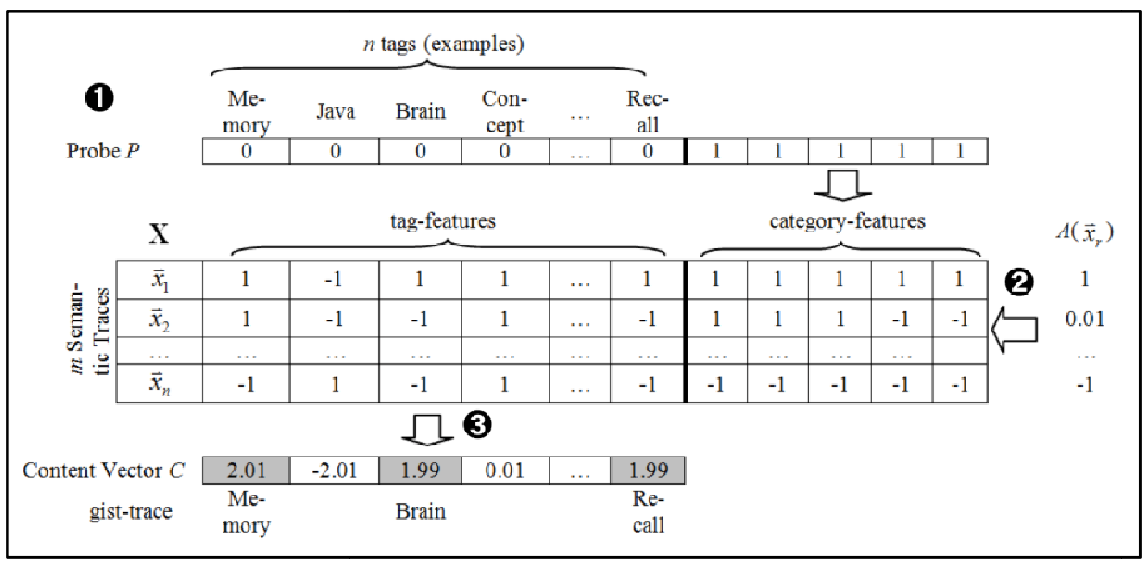

Fig. 1. Schematic presentation of the isTRM mechanics

One prerequisite to apply MINERVA2 is to group the semantic traces of a user into categories. In several social platforms, such as MENDELEY (www.mendeley.com), SemanticScuttle (www.semanticscuttle.sourceforge.net) or soboleo (www.soboleo.com), self-created folders or taxonomies complement the tagging functionality. In such environments, each folder or node of the taxonomy can be interpreted as a category cat. In more popular STS, such as Del.icio.us (www.delicious.com), some additional computational costs have to be invested to identify categories. The following paragraph provides a suggestion on how to group resources into categories.

Similar to the technique of collaborative filtering, the similarities between pairs of semantic traces, e.g. $\left(\underline{v}_{1}, x_{2}\right)$, can be computed by the cosine similarity measure (e.g. [4]). This measure can be applied to all pairs of semantic traces and a subsequent multidimensional scaling can represent these vectors as points in a multidimensional space. All pairs of traces whose Euclidean distance $d$ does not exceed a critical threshold $\tau$ can be assigned to the same category $c a t_{i}$. Each vector $\vec{x}_{r}$ needs a "label" indicating its category membership. Therefore, we extend each semantic trace by $o$ (so called) category-features $t=n+1 \ldots n+o$, representing the category to which resource $r$ belongs. For simplicity, in the example of Fig.1 there are only five category-features (i.e. $o=5$ ), which would allow for $2^{5}$ differentiations. For instance, the semantic trace $\vec{x}_{1}$ is labeled by the sequence $[1,1,1,1,1]$.

\subsection{Extracting the Gist of a User's Tag-Assignments}

After a new resource $r_{\text {new }}$, has been assigned to a category, e.g. cat $_{1}$, the isTRM starts by generating a probe $P$ (circled " 1 " in Fig. 5). The purpose of $P$ is to activate those semantic traces in the matrix $\mathbf{X}$, which belong to the same or similar category as the resource $r_{n e w} . P$ is also a vector with tag-features $\left[p_{t}=1 \ldots n\right]$ and category-features $\left[p_{t}=n+1 \ldots n+o\right]$ and bears the same label (category-features) as the resource $r_{n e w}$ $(1,1,1,1,1$ in the example of Fig.1); its tag-features are set at 0 . A particular 
MINERVA2 equation yields the similarity $S\left(\vec{x}_{r}\right)$ between $P$ and a semantic trace $\vec{x}_{r}$ by:

$$
S\left(\vec{x}_{r}\right)=\left(1 / N_{R}\right) \sum_{t=1}^{n} p_{t} x_{r t} .
$$

$N_{R}$ is the number of features for which either $p_{t}$ or $x_{r t}$ is nonzero. Since $S\left(\vec{x}_{r}\right)$ acts in a similar way as the Pearson correlation coefficient, the value of $S\left(\vec{x}_{r}\right)$ will be positive and high (approaching +1$)$ for all traces bearing the same or a similar label as $P\left(\vec{x}_{1}\right.$ in the example of Fig.1). The extent to which $P$ activates the trace $\vec{x}_{r}$ depends on a nonlinear function of $S\left(\vec{x}_{r}\right)$ given by $A\left(\vec{x}_{r}\right)=S\left(\vec{x}_{r}\right)^{3}$. Raising $S\left(\vec{x}_{r}\right)$ to the power 3 has proved to increase the activation differences between similar and less similar traces (see [3]).

To derive tag-recommendations from the matrix a content Vector $C$ with contentfeatures $c_{t}$ is computed summarizing the activation pattern across the matrix (circled " 3 " in Fig.1). The activation of each trace $A\left(\vec{x}_{r}\right)$ is multiplied by each of the trace's feature $x_{r t}$ (circled " 2 " in Fig.1). Then, these products are summed over traces:

$$
c_{t}=\sum_{r=1}^{m} A\left(\vec{x}_{r}\right) x_{r t} .
$$

The $c_{t}$-values indicate, "which features [in our case tags] are shared by the strongly activated traces" [3] and therefore, which tags belong to a prototypical tag combination of a user. In the example of Fig.1 the tags "memory", "brain" and "recall" constitute such a prototypical tag combination. Finally, we need a simple rule selecting an appropriate subset of tags for the gist-trace, i.e. the final tag recommendations. If the parameter $l$ specifies the number of tags to be selected, an appropriate subset is given by gist-trace $:=\left\{c_{t} \in C \mid \operatorname{rank}\left(c_{t}\right) \leq l\right\}$.

The isTRM is also conceived to mediate social sensemaking by identifying neighborhoods of users with similar categorization behavior. That could be realized by combining collaborative filtering with the content vector $C$. Referring to [4] the $k$ most similar users to user $u$ can be computed by:

$$
N_{u}^{k}:=\underset{v \in U \backslash\{\mathrm{u}\}}{\arg \max } \operatorname{sim}\left(C_{u}, C_{v}\right),
$$

where $\operatorname{sim}\left(C_{\mathrm{u}}, C_{\mathrm{v}}\right)$ is the cosine similarity between two vectors, in our case content vectors of the users $u$ and $v$. We assume that the neighborhood of user $u$ based on content vectors is a valid measure for user recommendations from a semantic memory perspective.

\section{Summary and Conclusion}

In this paper we introduced the isTRM, an implicit tag recommendation mechanism for the suggestion of psychologically plausible tag combinations and the identification 
of users with similar categorization behavior. It is based on empirical research on ST, built upon the memory theory MINERVA2 and treats users' TAS as verbalized semantic traces. The outcome of the isTRM is a gist-trace representing a tag combination that is assumed to resonate with the user's implicit semantic memory and thus, to give an appropriate categorical schema during the tagging activity, as suggested by [5]. By incorporating collaborative filtering, the isTRM appears to be a psychologically valid service to mediate social sensemaking within social learning environments.

In the near future, we aim at evaluating the isTRM. We will conduct an empirical study where different groups of participants will be supported by conventional TRMs as well as by the isTRM. On the one hand we will measure group differences with respect to the acceptance ratio, operationalized by the variables recall and precision (see [4]). On the other hand we will investigate the impact of the isTRM) on social sensemaking, operationalized by tag-quality (e.g. semantic distinctiveness) and resource-quality (e.g. coverage of different categories of the knowledge domain).

\section{References}

1. Fu, W.-T., Kannampallil, T.G., Kang, R.: A semantic imitation model of social tag choices. In: Proceedings of CSE 2009, pp. 66-73. ACM Press, New York (2009)

2. Gupta, M., Li, R., Yin, Z., Han, J.: Survey on social tagging techniques. In: 17th ACM SIGKDD, pp. 58-72. ACM Press, New York (2010)

3. Hintzman, D.L.: MINERVA 2: a simulation model of human memory. Behav. Res. Meth. Ins. C. 16, 96-101 (1984)

4. Jäschke, R., Marinho, L., Hotho, A., Schmidt-Thieme, L., Stumme, G.: Tag recommendations in social bookmarking systems. AI Commun. 21, 231-247 (2008)

5. Kuhn, A., Cahill, C., Quintana, C., Schmoll, S.: Using tags to encourage reflection and annotation on data during nomadic inquiry. In: Proceedings of CHI 2011, pp. 667-670. ACM Press, New York (2011)

6. Seitlinger, P., Ley, T.: Implicit imitation in social tagging: familiarity and semantic reconstruction. In: Proceedings of CHI 2012, pp. 1631-1640. ACM Press, New York (2012)

7. Seitlinger, P., Ley, T.: Implicit and explicit memory in social tagging: evidence from a process dissociation procedure. In: Proceedings of ECCE 2011, pp. 97-104. ACM Press, New York (2011) 